Pacific Journal of Mathematics

AN ESTIMATE OF INFINITE CYCLIC COVERINGS AND KNOT 


\title{
AN ESTIMATE OF INFINITE CYCLIC COVERINGS AND KNOT THEORY
}

\author{
Akio Kawauchi and Takao Matumoto
}

In this paper we estimate the homology torsion module of an infinite cyclic covering space of an $n$-manifold by the homology of a Poincaré duality space of dimension $n-1$. To be concrete, we apply it to knot theory. In particular, it follows that any ribbon $n$-knot $K \subset S^{n+2}(n \geqq 3)$ is unknotted if $\pi_{1}\left(S^{n+2}-\right.$ $K) \cong Z$. We add also in this paper a somewhat geometric proof to this unknotting criterion.

1. Statements of results. Let $X$ be a compact, connected and smooth, piecewise-linear or topological $n$-manifold with nonzero 1st Betti number, i.e., $H^{1}(X ; Z) \neq 0$. Let $\widetilde{X}$ be an infinite cyclic connected cover of $X$, that is, the cover of $X$ associated with an indivisible element of $H^{1}(X ; \boldsymbol{Z})$. We denote by $\langle t\rangle$ the covering transformation group of $\widetilde{X}$ with a specified generator $t$. Let $F$ be a field and $F\langle t\rangle$ be the group algebra of $\langle t\rangle$ over $F$. For $H_{*}=$ $H_{*}(\tilde{X} ; F)$ or $H_{*}(\tilde{X}, \partial \tilde{X} ; F), H_{*}$ is canonically regarded as an $F\langle t\rangle-$ module. We define $T_{*}=\operatorname{Tor}_{F\langle t\rangle} H_{*}$ and $T^{*}=\operatorname{Hom}_{F}\left[T_{*}, F\right]$. We assume $\tilde{X}$ is $F$-orientable. Note that $T_{0}(\tilde{X} ; F)=H_{0}(\tilde{X} ; F) \cong F$ and $T_{n-1}(\tilde{X}, \partial \tilde{X} ; F) \cong F$. (Cf. [5, Duality Theorem (II) and Remark 1.3].) Let $M$ be a connected Poincaré duality space with boundary $\partial M$ of dimension $n-1$ over $F$.

THEOREM. Suppose there is a map $f:(M, \partial M) \rightarrow(\widetilde{X}, \partial \tilde{X})$ such that $f_{*} H_{n-1}(M, \partial M ; F)=T_{n-1}(\tilde{X}, \partial \widetilde{X} ; F)$. Then

$$
\operatorname{dim}_{F} H_{q}(M ; F) \geqq \operatorname{dim}_{F} T_{q}(\widetilde{X} ; F)
$$

for all q. Further, if $f_{*} H_{q}(M ; F) \subset T_{q}(\widetilde{X} ; F)$ for some $q$, then $f_{*} H_{q}(M ; F)=T_{q}(\widetilde{X} ; F)$. In particular, if $T_{q}(\widetilde{X} ; F)=H_{q}(\tilde{X} ; F)$ (e.g., $\left.H_{q}(X ; F) \cong H_{q}\left(S^{1} ; F\right)\right)$ for some $q$, then the homomorphism

$$
f_{*}: H_{q}(M ; F) \longrightarrow H_{q}(\widetilde{X} ; F)
$$

is onto.

Note 1. Our proof will imply also that

$$
\operatorname{dim}_{F} H_{n-q-1}(M, \partial M ; F) \geqq \operatorname{dim}_{F} T_{n-q-1}(\tilde{X}, \partial \widetilde{X} ; F)
$$

for all $q$ and, if $f_{*} H_{n-q-1}(M, \partial M ; F) \subset T_{n-q-1}(\tilde{X}, \partial \tilde{X} ; F)$ for some $q$, then $f_{*} H_{n-q-1}(M, \partial M ; F)=T_{n-q-1}(\widetilde{X}, \partial \widetilde{X} ; F)$.

In case $X$ is oriented and piecewise-linear and $\tilde{X}$ is obtained 
from a piecewise-linear map $g: X \rightarrow S^{1}$, the preimage $X_{1}=g^{-1}(p)$ is a bicollared, oriented, proper $(n-1)$-submanifold of $\widetilde{X}$ for any nonvertex point $p$ of $S^{1}$. Then, we see that the inclusion $i:\left(X_{1}, \partial X_{1}\right) \subset$ $(\tilde{X}, \partial \widetilde{X})$ sends the fundamental class of $X_{1}$ to a generator of $T_{n-1}(\tilde{X}, \partial \widetilde{X} ; F)$ for any $F$. [Proof. Let $X^{\prime}$ be a manifold obtained from $X$ by splitting along $X_{1} . \quad X^{\prime}$ is imbedded canonically in $\tilde{X}$ so that $\partial X^{\prime}=X_{1} \cup\left(X^{\prime} \cap \partial X\right) \cup-t X_{1}$. This implies that $(1-t)\left[X_{1}\right]=$ $\left[X_{1}\right]=t\left[X_{1}\right]=0$ in $H_{n-1}(\widetilde{X}, \partial \widetilde{X} ; F)$, i.e., $\left[X_{1}\right] \in T_{n-1}(\widetilde{X}, \partial \widetilde{X} ; F) . \quad\left[X_{1}\right] \neq$ 0 in $H_{n-1}(X, \partial X ; F)$ and hence in $H_{n-1}(\tilde{X}, \partial \tilde{X} ; F)$, since it is the Poincaré dual of $g^{*}\left[S^{1}\right] \in H^{1}(X ; F)$. Thus, $\left[X_{1}\right]$ generates $T_{n-1}(\tilde{X}, \partial \tilde{X}$; $F) \cong F$.] Let $\hat{X}_{1}$ be the interior oriented connected sum of the components of $X_{1}$. Since $\tilde{X}$ is connected, we can construct from $i$ a map $\hat{i}:\left(\hat{X}_{1}, \partial \hat{X}_{1}\right) \rightarrow(\tilde{X}, \partial \widetilde{X})$ such that $\hat{i}_{*} H_{n-1}\left(\hat{X}_{1}, \partial \hat{X}_{1} ; F\right)=T_{n-1}(\tilde{X}$, $\partial \widetilde{X} ; F)$. From this observation and the theorem, we see the following:

CoRollaRy 1. $\operatorname{dim}_{F} H_{q}\left(X_{1} ; F\right) \geqq \operatorname{dim}_{F} T_{q}(\tilde{X} ; F)$ for all $q$ and $F$. If $i_{*} H_{q}\left(X_{1} ; F\right) \subset T_{q}(\widetilde{X} ; F)$ for some $q$ and some $F$, then $i_{*} H_{q}\left(X_{1}\right.$; $F)=T_{q}(\widetilde{X} ; F)$.

In knot theory this corollary gives a general relation between the homology of a Seifert manifold of a knot (or link) and its knot (or link) module (associated with an infinite cyclic covering). For a classical knot (i.e., 1-knot) $k$, this has been recognized as (the genus of $k) \geqq(1 / 2) \cdot($ the degree of the knot polynomial of $k$ ). (Cf. H. Seifert [9].)

Next, suppose $X$ is orientable and $H_{1}(X ; \boldsymbol{Z}) \cong \boldsymbol{Z}$. Such a manifold occurs, for example, as the complement of an open regular neighborhood of a closed connected orientable $(n-2)$-manifold imbedded piecewise-linearly in $S^{n+2}$. By Poincaré duality $H_{n-1}(X$, $\partial X ; \boldsymbol{Z}) \cong \boldsymbol{Z}$.

CoROLlary 2. If there is a map $f:(M, \partial M) \rightarrow(X, \partial X)$ inducing an isomorphism $f_{*}: H_{n-1}(M, \partial M ; \boldsymbol{Z}) \cong H_{n-1}(X, \partial X ; \boldsymbol{Z})$ and a 0 -map $f_{*}=0: H_{1}(M ; \boldsymbol{Z}) \rightarrow H_{1}(X ; \boldsymbol{Z})$, then

$$
\operatorname{dim}_{F} H_{q}(M ; F) \geqq \operatorname{dim}_{F} T_{q}(\widetilde{X} ; F)
$$

for all $q$ and $F$.

To see this, note that $H_{n-1}(\tilde{X}, \partial \widetilde{X} ; \boldsymbol{Z}) \cong \boldsymbol{Z}$ and $t$ acts trivially on it and the covering projection $\tilde{X} \rightarrow X$ induces an isomorphism $H_{n-1}(\tilde{X}, \partial \tilde{X} ; \boldsymbol{Z}) \cong H_{n-1}(X, \partial X ; \boldsymbol{Z})$. This follows from [3, Theorem 2.3], (or its topological version [4]) and the Wang exact sequence. So, it suffices to show that $f$ has a lifting to $\tilde{X}$. This is clear, 
however, by the assumption that $f_{*}: H_{1}(M ; Z) \rightarrow(X ; Z)$ is a 0 -map.

For the following application, spaces and maps are considered in the piecewise-linear category. Let $L$ be a trivial $n$-link in $S^{n+2}$ of some $r+1$ components and a collection $\left\{B_{1}, \cdots, B_{r}\right\}$ of $r(n+1)$ balls imbedded locally-flatly and mutually disjointly in $S^{n+2}$ such that for each $i B_{i}$ spans $L$ as 1-handle i.e., $B_{i} \cap L=\left(\partial B_{i}\right) \cap L=$ the disjoint union of two $n$-balls. An $n$-knot $K$ in $S^{n+2}$ is called a ribbon $n$-knot if it is obtained from such an $L$ and a $\left\{B_{1}, \cdots, B_{r}\right\}$ by doing an imbedded surgery. (Cf. T. Yanagawa [12], R. Hitt [1].) The knot $K$ is often said to be a fusion of the link $L$ along 1-handles $\left\{B_{1}, \cdots, B_{r}\right\}$.

CoROllary 3. Let $n \geqq 3$. A ribbon n-knot $K$ is unknotted, if $\pi_{1}\left(S^{n+2}-k\right) \cong Z$.

To see this, note that any ribbon $n$-knot has a Seifert $(n+1)$ manifold $M$, homeomorphic to a manifold of the form $\#^{m} S^{1} \times S^{n}$ Int $B^{n+1}\left(B^{n+1}\right.$ is an $(n+1)$-ball.) ([12], [1]). Let $X=S^{n+2}$-Int $N(K)$, $N(K)$ being a regular neighborhood of $K$ in $S^{n+2}$. The manifold $X \cap M(\cong M)$ gives a generator of $H_{n+1}(X, \partial X ; Z)=Z$ and the inclusion $X \cap M \subset X$ induces a 0 -map on $H_{1}$. By Corollary $2, T_{i}(\tilde{X}$; $F)=0, i \neq 0,1, n$. (Of course, one can also apply Corollary 1 to obtain this.) But $T_{*}(\tilde{X} ; F)=H_{*}(\tilde{X} ; F)$. As a result, $\widetilde{H}_{*}(\widetilde{X} ; F)=0$ by using Milnor duality [8] or [5, Duality Theorem (II)], since $\tilde{X}$ is simply connected. Then by taking $F=\boldsymbol{Q}$, we see that $\widetilde{H}_{*}(\widetilde{X} ; \boldsymbol{Z})$ is a torsion group. Next, by taking $F=Z_{p}, p$ prime, and considering the universal coefficient theorem, the torsion product $\operatorname{Tor}_{z}\left(H_{*-1}(\tilde{X}\right.$; $\left.\boldsymbol{Z}), \boldsymbol{Z}_{p}\right)=0$. This shows that $\widetilde{H}_{*}(\tilde{X} ; \boldsymbol{Z})=0$ and $X$ has the homotopy type of $S^{1}$. By [6], [10], [11], $K$ is unknotted for $n \geqq 3$.

Note 2. For $n=2$, a corresponding result is proved by $\mathrm{Y}$. Marumoto [7] in the simplest case, that is, the case of $L$ having two components. However, a general case is unknown.

2. Proof of theorem. Let $i: T_{*} \subset H_{*}$. $i$ induces an epimorphism $i^{*}: H^{*} \rightarrow T^{*}$. Let $x \in H^{q}(\widetilde{X} ; F)$ such that $i^{*}(x) \neq 0$. By [5, Duality Theorem (II)], the cup product $H^{q}(\widetilde{X} ; F) \times H^{n-q-1}(\widetilde{X}, \partial \widetilde{X}$; $F) \stackrel{\cup}{\rightarrow} H^{n-1}(\tilde{X}, \partial \widetilde{X} ; F)$ induces a nonsingular pairing $T^{q}(\tilde{X} ; F) \times$ $T^{n-q-1}(\tilde{X}, \partial \tilde{X} ; F) \rightarrow T^{n-1}(\tilde{X}, \partial \tilde{X} ; F)$, also denoted by $U$. Hence we find an element $y \in H^{n-q-1}(\tilde{X}, \partial \widetilde{X} ; F)$ such that $i^{*}(x) \cup i^{*}(y)=i^{*}(x \cup$ $y) \neq 0$. By assumption, $f:(M, \partial M) \rightarrow(\widetilde{X}, \partial \widetilde{X})$ induces the following commutative triangle 


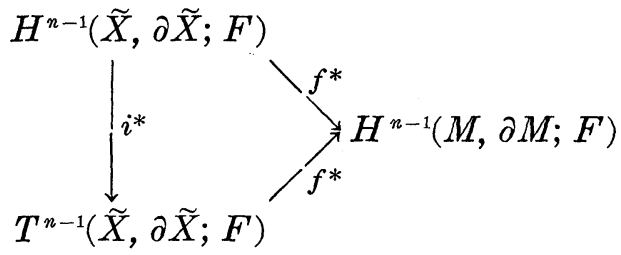

and $f^{*}: T^{n-1}(\widetilde{X}, \partial \widetilde{X} ; F) \rightarrow H^{n-1}(M, \partial M ; F)$ is an isomorphism. Thus, $f^{*}(x \cup y)=f^{*}(x) \cup f^{*}(y) \neq 0$, so that $f^{*}(x) \neq 0$. We obtain a (noncanonical) monomorphism $r: T^{q}(\tilde{X} ; F) \rightarrow H^{q}(M ; F)$. Hence, $\operatorname{dim}_{F} T_{q}(\widetilde{X}$; $F)=\operatorname{dim}_{F} T^{q}(\tilde{X} ; F) \leqq \operatorname{dim}_{F} H^{q}(M ; F)=\operatorname{dim}_{F} H_{q}(M . F)$. If $f_{*} H_{q}(M ; F) \subset$ $T_{q}(\tilde{X} ; F)$, then we may replace $r$ by a canonical epimorphism $r^{\prime}: T^{q}(\widetilde{X} ; F) \rightarrow \operatorname{Hom}_{F}\left[f_{*} H_{q}(M ; F), F\right]$ composed with the natural inclusion into $H^{q}(M ; F)$. Since $r^{\prime}$ is an isomorphism, we see that $f_{*} H_{q}(M ; F)=T_{q}(\widetilde{X} ; F)$. This completes the proof of the theorem.

3. Alternative proof of Corollary 3. We now describe a different, somewhat geometric proof of Corollary 3. This method, as a matter of fact, has been earlier obtained and is near to the argument of [2]. Let $T(m)$ be an $n$-manifold homeomorphic to $\#^{m} S^{1} \times S^{n-1}$ and imbedded locally-flatly in $S^{n+2}$. (The following four lemmas are true when $n \geqq 2$.) For $m=0, T(m)$ is an $n$-sphere, i.e., an $n$-knot. Such a $T(m)$ is unknotted if it bounds a manifold locally-flatly imbedded in $S^{n+2}$ and homeomorphic to a disk sum $G^{m} S^{1} \times B^{n}$. As an analogous argument to [2, Theorem 1.2], we have the following:

\subsection{Any two unknotted $T(m)_{1}, T(m)_{2}$ are ambient isotopic.}

Thus, the following is obtained:

3.2. If $T(m)$ is unknotted, $S^{n+2}-T(m)$ is homotopy equivalent to a bouquet $S^{1} \vee S^{2} \vee \cdots \vee S^{2} \vee S^{n} \vee \cdots \vee S^{n}$ of one 1-sphere, $m$ 2spheres and $m$ n-spheres. [Regard $T(m)$ as the common boundary of $\xi^{m} S^{1} \times B^{n}$ and $\natural^{m} B^{2} \times S^{n-1}$ whose union forms an unknotted $(n+1)$-sphere $S_{0}^{n+1}$ in $S^{n+2}$. Then, $S^{n+2}-T(m)$ is homotopy equivalent to the suspension of $S_{0}^{n+1}-T(m)$.]

3.3. Let $T(m+1)$ and $T(m+1)^{\prime}$ be the manifolds obtained from the same $T(m)$ by surgeries along 1-handles $B^{n+1}$ and $B^{\prime n+1}$ on $T(m)$ imbedded locally-flatly in $S^{n+2}$, respectively. If $\pi_{1}\left(S^{n+2}-\right.$ $T(m)) \cong Z$, then $T(m+1)$ and $T(m+1)^{\prime}$ are ambient isotopic.

This is proved easily as an analogy to [2, Lemma 2.7].

From 3.3 and the definition of ribbon knots, we see the following: 
3.4. For any ribbon $n$-knot $K$ obtained from $(m+1)$ balls and $m$ 1-handles, the surgery along some standard mutually disjoint $m$ 1-handles on $K$ imbedded locally-flatly in $S^{n+2}$ produces an unknotted $T(m)$. Further, if $\pi_{1}\left(S^{n+2}-K\right) \cong Z$, then $T(m)$ is ambient isotopic to a knot sum $K \# T(m)^{\prime}$ for some unknotted $T(m)^{\prime}$.

Now assume $\pi_{1}\left(S^{n+2}-K\right) \cong Z$. In 3.4 , let $E=S^{n+2}-K, X=$ $S^{n+2}-T(m)$ and $X^{\prime}=S^{n+2}-T(m)^{\prime}$. Take their infinite cyclic connected covers. We have $\widetilde{H}_{*}(\widetilde{E} ; \boldsymbol{Z}) \oplus \widetilde{H}_{*}\left(\widetilde{X}^{\prime} ; \boldsymbol{Z}\right) \cong \widetilde{H}_{*}(\widetilde{X} ; \boldsymbol{Z})$ as $\boldsymbol{Z}\langle t\rangle$ modules. By $3.2, \widetilde{H}_{*}\left(\widetilde{X}^{\prime} ; \boldsymbol{Q}\right)$ and $\widetilde{H}_{*}(\widetilde{X} ; \boldsymbol{Q})$ are free $\boldsymbol{Q}\langle t\rangle$-modules of the same rank, so that $\widetilde{H}_{*}(\widetilde{E} ; \boldsymbol{Q})=0$, i.e., $\widetilde{H}_{*}(\widetilde{E} ; \boldsymbol{Z})$ is a torsion group. By 3.2 again, $\widetilde{H}_{*}\left(\widetilde{X}^{\prime} ; \boldsymbol{Z}\right)$ and $\widetilde{H}_{*}(\widetilde{X} ; \boldsymbol{Z})$ are free abelian, hence $\widetilde{H}_{*}(\widetilde{E} ; \boldsymbol{Z})=0$ and $E$ has the homotopy type of $S^{1}$. By [6], [10], [11], $K$ is unknotted for $n \geqq 3$.

\section{REFERENCES}

1. L. R. Hitt, Characterization of ribbon n-knots, Notices Amer. Math. Soc., 26 (1979), A-128.

2. F. Hosokawa and A. Kawauchi, Proposals for unknotted surfaces in four-spaces, Osaka J. Math., 16 (1979), 233-248.

3. A. Kawauchi, A partial Poincaré duality theorem for infinite cyclic coverings, Quart. J. Math., 26 (1975), 437-458.

4. - A partial Poincare duality theorem for topological infinite cyclic coverings and applications to higher dimensional topological knots, (unpublished).

5. — On quadratic forms of 3-manifolds, Invent. Math., 43 (1977), 177-198.

6. J. Levine, Unknotting spheres in codimension two, Topology, 4 (1966), 9-16.

7. Y. Marumoto, On ribbon 2-knots of 1-fusion, Math. Sem. Notes Kobe Univ., 5 (1977), 59-68.

8. J. W. Milnor, In finite cyclic coverings, Conference on the Topology of Manifolds edited by Hocking, Prindle, Weber and Schmidt, Boston, Mass., 1968, 115-133.

9. H. Seifert, Über das Geschlecht von Knoten, Math. Ann., 110 (1934), 571-592.

10. J. L. Shaneson, Embeddings with codimension two of spheres in spheres and H-cobord. disms of $S^{1} \times S^{3}$, Bull. Amer. Math. Soc., 74 (1968), 972-974.

11. J. Stallings, On topologically unknotted spheres, Ann. of Math., 77 (1963), 490-503

12. T. Yanagawa, On ribbon 2-knots, Osaka J. Math., 6 (1969), 447-464.

Received September 25, 1979. The authors are both supported in part by National Science Foundation grants.

The Institute for Advanced Study

Princeton, NJ 08540

Current address of the second author: Department of Mathematics, Hiroshima University Hiroshima 730, Japan 



\section{PACIFIC JOURNAL OF MATHEMATICS}

\section{EDITORS}

DONALD BABBITT (Managing Editor)

University of Galifornia

Los Angeles, California 90024

HUGO RossI

University of Utah

Salt Lake City, UT 84112

C. C. MOORE AND ANDREW OGG

University of California

Berkeley, CA 94720
J. DUGUNDJI

Department of Mathematics University of Southern California Los Angeles, California 90007

R. FINN AND J. MILGRAM Stanford University

Stanford, California 94305

ASSOCIATE EDITORS

E. F. BECKENBACH

B. H. NeumanN

F. WOLF

K. YosHidA

\section{SUPPORTING INSTITUTIONS}

UNIVERSITY OF BRITISH COLUMBIA UNIVERSITY OF SOUTHERN CALIFONIA CALIFORNIA INSTITUTE OF TECHNOLOGY UNIVERSITY OF CALIFORNIA MONTANA STATE UNIVERSITY STANFORD UNIVERSITY UNIVERSITY OF HAWAII UNIVERSITY OF NEVADA, RENO UNIVERSITY OF TOKYO U'NIVERSITY OF UTAH NEW MEXICO STATE UNIVERSITY WASHINGTON STATE UNIVERSITY OREGON STATE UNIVERSITY UNIVERSITY OF OREGON UNIVERSITY OF WASHINGTON 


\section{Pacific Journal of Mathematics}

\section{Vol. 90, No. $1 \quad$ September, 1980}

Shashi Prabha Arya and M. K. Singal, On the locally countable sum

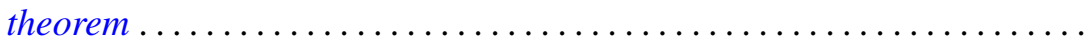

John Theodore Baldwin and David William Kueker, Ramsey quantifiers and

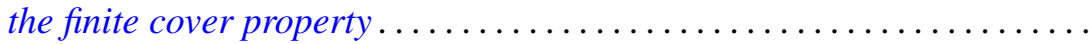

Richard Body and Roy Rene Douglas, Unique factorization of rational

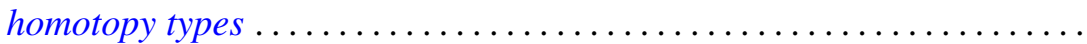

Ethan Bolker and Ben G. Roth, When is a bipartite graph a rigid

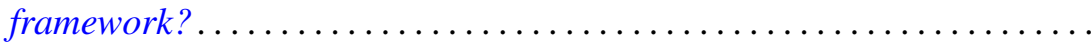

Alicia B. Winslow, Continua in the Stone-Čech remainder of $R^{2} \ldots \ldots \ldots$

Richard D. Carmichael and Elmer Kinji Hayashi, Analytic functions in tubes which are representable by Fourier-Laplace integrals ..............

Stephen D. Cohen, The Galois group of a polynomial with two indeterminate coefficients ..............................

Russell Allan Johnson, Strong liftings commuting with minimal distal

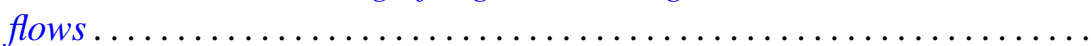

Elgin Harold Johnston, The boundary modulus of continuity of harmonic

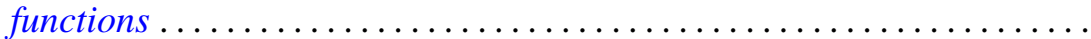

Akio Kawauchi and Takao Matumoto, An estimate of infinite cyclic

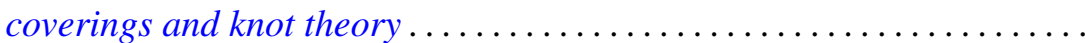

Keith Milo Kendig, Moiré phenomena in algebraic geometry: rational alternations in $\mathbf{R}^{2}$...

Roger T. Lewis and Lynne C. Wright, Comparison and oscillation criteria for selfadjoint vector-matrix differential equations .

Teck Cheong Lim, Asymptotic centers and nonexpansive mappings in conjugate Banach spaces .......................

David John Lutzer and Robert Allen McCoy, Category in function spaces.

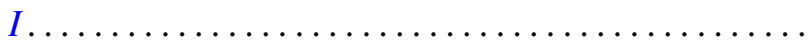

Richard A. Mollin, Induced p-elements in the Schur group ...

Jonathan Simon, Wirtinger approximations and the knot groups of $F^{n}$ in $S^{n+2}$

Robert L. Snider, The zero divisor conjecture for some solvable groups...

H. M. (Hari Mohan) Srivastava, A note on the Konhauser sets of biorthogonal polynomials suggested by the Laguerre polynomials...

Nicholas Th. Varopoulos, A probabilistic proof of the Garnett-Jones theorem on BMO.

Frank Arvey Wattenberg, $[0, \infty]$-valued, translation invariant measures on $N$ and the Dedekind completion of ${ }^{*} R \ldots \ldots \ldots \ldots . .$. 